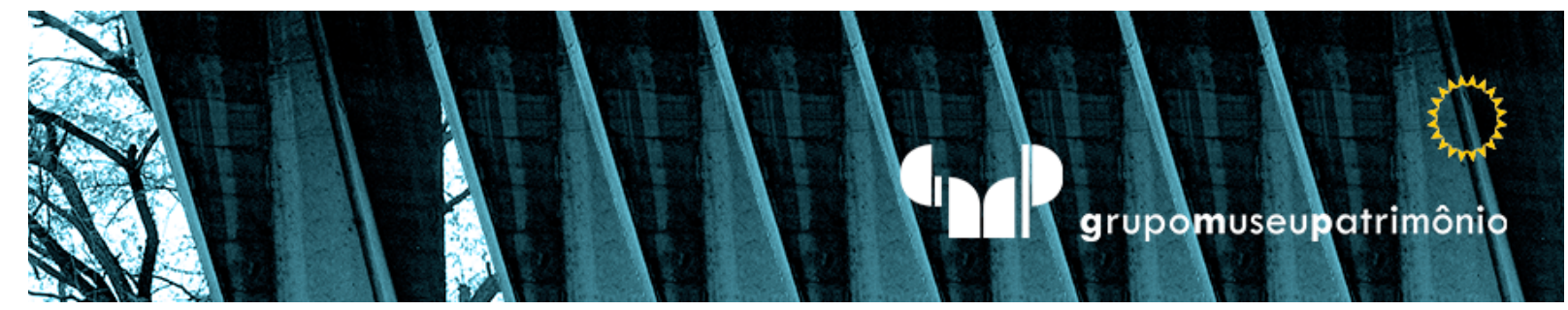

\title{
Museus e Desenvolvimento Sustentável
}

\author{
Museos y desarrollo sostenible
}

\section{Museums and Sustainable Development}

Mariana Espel de Oliveira,

Pós Graduada em Património Cultural e Museologia pela Universidade de Coimbra e em Ciência Política pela Universidade de Aveiro. Mestranda em Museologia na Faculdade de Letras da Universidade do Porto, Porto, Portugal.mraeo@outlook.pt 


\section{Resumo}

O presente estudo propõe um plano metodológico elaborado com base nos Indicadores de Sustentabilidade sugeridos e publicados pela Organização de Cooperação e de Desenvolvimento Económico/OCDE em 2018. O objetivo é de reconhecer quais são os outputs gerados pelas atividades de Museus em Portugal que contribuem com a Agenda 2030 do Desenvolvimento Sustentável da Organização das Nações Unidas/ONU. O resultado demonstrou uma efetiva possibilidade de medição dos níveis de sustentabilidade dos museus e a potencialidade dessas instituições em colaborar com esta Agenda.

Palavras-Chave: Desenvolvimento Sustentável, Museus, Planejamento Estratégico, OCDE, ONU.

\section{Resumen}

El presente estudio propone un plan metodológico elaborado en base a los Indicadores de sostenibilidad sugeridos y publicados por la Organización para la Cooperación y el Desarrollo Económico/OCDE en 2018. El objetivo es reconocer qué resultados de las actividades de los museos en Portugal contribuyen a la Agenda 2030 de las Naciones Unidas para el Desarrollo Sostenible. El resultado demostró una posibilidad efectiva de medir los niveles de sostenibilidad del museo y el potencial de estas instituciones para colaborar con esta Agenda.

Palavras-Clave: Desarrollo sostenible, museos, planificación estratégica, OCDE, ONU.

\section{Abstract}

The present study proposes a methodological plan prepared based on the Sustainability Indicators suggested and published by the Organization for Economic Cooperation and Development/ OECD in 2018. The objective is to recognize which outputs are generated by the activities of Museums in Portugal that contribute to the UN Sustainable Development 2030 Agenda. The result demonstrated an effective possibility of measuring the sustainability levels of museums and the potential of these institutions to collaborate with this Agenda.

Keywords: Sustainable Development, Museums, Strategic Planning, OECD, UN. 


\section{INTRODUÇÃO}

E ste estudo ${ }^{1}$ tem o objetivo de demonstrar a importância de se reconhecer os fatores existentes nas atividades dos museus que contribuem para o Desenvolvimento Sustentável da Sociedade.

Para reconhecer a mais-valia destas instituições e envolvê-las em programas que objetivam a implementação de Políticas Públicas para o Desenvolvimento Sustentável (DS) - é necessário que primeiro se reconheça quais sãos as atividades, ações serviços e outputs gerados pelos museus à serem utilizados de forma efetiva em futuros programas que objetivam contribuir para o DS.

Diante da necessidade observada, este estudo pretende ser um contributo inicial para que os museus em Portugal tenham diretivas que auxiliem no reconhecimento de outputs que colaboram com os pilares do DS. Aplicando este procedimento é compreensível que estas instituições terão condições e

1 Este estudo foi parcialmente apresentado em maio de 2019 no Departamento de Ciências Sociais e Políticas do Território na Universidade de Aveiro, Portugal para conclusão da Disciplina Ambiente e Estratégias do Desenvolvimento no âmbito do Curso de Mestrado em Ciência Política, primeiro ano, segundo semestre, sob tutoria dos Professores Doutores Artur Rosa Pires e José Manuel Martins. 
informação concreta para apresentar ao Poder Público formas de atuação como um agente dinamizador do DS.

Para atingir o objetivo delineado nos parágrafos anteriores, o estudo propõe um método para que seja feita a observação de outputs gerado pelos Museus. O modelo de recolha de dados está orientado pelas diretrizes da OCDE, publicado em Novembro de 2018, no Guia "Culture and local Development: Maximising the Impact -Launch version Guide for Local Governments, Communities and Museums".

Os dois museus que serviram de referência e como objetos de estudo para observação estão situados na Região Centro de Portugal. A recolha de dados possibilitou o reconhecimento das aptidões de cada museu participante.

A escolha por Portugal, considerou o fato do País ser membro da Organização das Nações Unidas (ONU), o que traz de forma inevitável, o compromisso de elaborar Políticas Públicas (PP) relacionadas a Agenda 2030 para o DS. Outro aspeto considerado está relacionado a inexistência de diretrizes para recolha e análise desta tipologia de dados.

Neste estudo, foram realizadas pesquisas bibliográficas que contemplam a conceitualização do Planejamento Estratégico relacionado ao Desenvolvimento Sustentável, e foram feitas também, consultas em publicações que relacionam Museus e o conceito de DS.

Na segunda fase do estudo, foram realizadas observações e aplicação de questionários em duas instituições museológicas da Região Centro de Portugal com o objetivo de reconhecer se os Parâmetros OCDE previamente organizados, eram reconhecidos nas instituições mencionadas.

O trabalho de investigação realizado está estruturado em cinco itens, iniciando pela Revisão de Literatura que está organizada em dois subitens, denominados Planejamento Estratégico e Desenvolvimento Sustentável e o segundo subitem denominado Museus e Desenvolvimento Sustentável. O segundo item, denominado Desenvolvimento, contempla o processo metodológico realizado 
neste trabalho. Os resultados advindos do processo de investigação realizado por meio do método de Estudo de Caso (multi casos), estão delienados no terceiro item. No quarto item, foi acrescida uma breve demonstração de como os indicadores elaborados para recolha de dados, correspondem aos objetivos da Agenda 2030 para o DS da ONU. Por fim, no último item denominado Considerações finais, serão elencados aspectos observados durante a elaboração deste estudo, onde se pretende delinear as descobertas, implicações e pertinência deste trabalho

\section{Objetivo e justificativa do estudo}

O objetivo do estudo é oferecer aos Museus em Portugal um contributo inicial, para que estas instituições possam reconhecer quais são os outputs gerados nas suas atividades, que poderão contribuir com o DS da localidade em que o Museu está inserido.

A relevância para elaboração do estudo está relacionado ao protagonismo dos Museus nas Agendas relacionadas ao DS. Desta forma, é necessário que estas instituições tenham dados organizados para demonstrar e reconhecer a sua efetiva colaboração e também, elaborar possíveis formas de cooperar com programas relacionados ao DS. Sem isto, dificilmente suas ações serão reconhecidas e apoiadas pelo Poder Público.

Segundo Pires (2012) mensurar e reconhecer o nível de sustentabilidade das instituições é "uma excelente forma de comunicar, sensibilizar e educar, não só a população em geral, mas também políticos, decisores, técnicos e os mais variados agentes econômicos, para a necessidade de alterar estilos de vida e comportamentos" (2012: p. 12).

Em suma, este método se demonstra relevante pelo fato deste disponibilizar ao setor governamental, informação credível que demonstre a efetiva colaboração da instituição na Agenda do DS, tendo assim meios que evidenciem o que realmente o setor Público pode extrair de positivo e utilizar de forma estratégica para o DS da sociedade (Wickham and Lehman, 2015: p. 4). 
Com o reconhecimento do objetivo e justificativa do estudo, o item seguinte irá abordar de forma breve, a base teórica que serviu de referência para a conceitualização e elaboração deste estudo.

\section{Revisão de Literatura}

A Agenda 2030 da da ONU reconhece o Museu como um relevante ator institucional colaborador dos programas de Políticas Públicas relacionadas ao cumprimento da Agenda $2030^{2}$ da ONU. (Unesco,2018).

Para implementação de PP relacionadas ao cumprimento desta Agenda, é de suma importância que a administração local, regional e nacional, realizem Planos Estratégicos para definir diretrizes que objetivam atingir as orientações da ONU para o DS. No entanto, é possível facilitar o processo de incluisão dos museus nestes planejamentos com a realização de um estudo aprofundado que aborde quais as atribuições que tal organização é capaz de suportar, para então ser submetida como um agente do DS.

Atualmente na literatura, foram identificados estudos que delimitam as possíveis intervenções e contribuições que poderão ser atribuídas aos museus em relação ao cumprimento dos objetivos da agenda 2030. Como exemplo, o Guia publicado em 2018 pela Organização para a Cooperação e Desenvolvimento Económico (OCDE) e Comissão Internacional dos Museus (ICOM), denominado "Culture and local Development: Maximising the Impact Launch version Guide for Local Governments, Communities and Museums". Este guia evidencia quais as atividades e modos de operação existentes nos

\footnotetext{
${ }^{2}$ «A Agenda 2030 para o Desenvolvimento Sustentável da Organização das Nações Unidas é constituída por 17 Objetivos de Desenvolvimento Sustentável (ODS) e foi aprovada em setembro de 2015 por 193 membros. Os ODS resultam do trabalho conjunto de governos e cidadãos de todo o mundo para criar um novo modelo global para acabar com a pobreza, promover a prosperidade e o bem-estar de todos, proteger o ambiente e combater as alterações climáticas». Fonte: https://www.ods.pt/ods/
} 
museus, que estão diretamente relacionadas aos objetivos da Agenda 2030 do DS e enfatiza a contribuição da instituição como um dinamizador nas comunidades locais.

Ademais, este guia e outras bases bibliográficas referenciais também apontam estudos empíricos que demonstram a real representatividade dos museus como contribuidores do DS, no entanto, não foram localizados na literatura, trabalhos que organizam tais parâmetros para o cenário dos museus em Portugal.

Para contribuir com esta lacuna, a proposta é realizar, com base nas diretrizes da OCDE, uma observação em dois museus portugueses para assim, verificar se tais parâmetros são passíveis de serem utilizados no ambiente. Somado a isto, verificar se essas instituições já contêm estas características. Este estudo intenta ser um primeiro contributo para criação de um roteiro, que auxilie as lideranças governamentais nacionais, regionais e das localidades, a distinguir o espectro de oportunidades existentes nestas instituições.

Sendo assim, será delineado a seguir, um breve espectro conceitual para auxiliar na compreensão da interligação de temas como Planeamento Estratégico e DS, museus locais e DS, para então seguir com a fase prática deste estudo.

\section{Planejamento Estratégico e Desenvolvimento Sustentável}

O projeto de DS definido em 1987 pelo relatório Nosso Futuro Comum de Brundtland exibiu uma série de diretrizes ao mundo, objetivando a criação de programas governamentais que conduzissem a um caminho comum, o desenvolvimento econômico mundial ordenado, que corrigisse disparidades entre as diferentes nações somado a proteção dos recursos limitados existentes no planeta (NFC, 1991, p.46).

Neste primeiro momento o conceito definia que "Um Futuro Comum" só seria alcançado com a devida participação das nações consideravelmente prósperas, e que deveria ser contemplado em seus processos políticos um desenvolvimento baseado nos Pilares Econômicos, Ambientais e Sociais. 
O conceito de DS segundo Nascimento (2012, p. 51) é compreendido em duas diferentes vertentes. A primeira está relacionada a disciplina de Biologia que se refere a capacidade "de recuperação e reprodução do ecossistema em face as agressões antrópicas ou naturais". Já na segunda vertente, a conceptualização abarca o conceito econômico. Esta conceptualização é a que mais se aproxima do conceito do DS defendido no relatório de Bruntdland de 1987 (Nosso Futuro Comum).

De forma breve é possível compreender que o conceito defende a necessidade da prosperidade das nações ser delineado de forma regulada, a ponto que o processo econômico respeite as questões ambientais de escassez e de impactos negativos nos recursos naturais finitos e acate as questões sociais que rodeiam e sofrem impacto diante do processo de Desenvolvimento Econômico. Com esta formulação, o conceito exprime o objetivo de resguardar às gerações futuras, condições básicas para sobreivência e melhoria das condições de vida em países subdesenvolvidos.

A cronologia do conceito é marcada pelo início da elaboração do Relatório Nosso Futuro Comum, divulgado formalmente às nações na Cimeira ocorrida no Rio de Janeiro - Brasil em 1992, conhecida como ECO`92. Nesta cimeira foi lançado o primeiro guia de diretrizes para o DS e foram também, enumerados os países signatários desta Agenda. Definido os rumos para os próximos anos, a Agenda orientada pela ONU, teve como base a criação de orientações para a elaboração de PP que contemplasse o DS que deveriam ser seguidas até 2015, definindo assim a Agenda 21.

Em 2015, foi realizada a última cimeira em Paris, organizada pela ONU onde foram discutidas as respostas obtidas da implantação da Agenda 21 e elaborada uma nova fase, denominada Agenda 2030, onde foram elencadas as necessidades e o cenário da sociedade global do tempo presente e as lacunas deixadas no programa passado.

Na obra denominada Sustainable development in a post-Brundtland world (2006), os autores defendem que após dezoito anos da conceitualização do 
termo DS, a sua aplicação ainda apresenta divergência no seu entendimento. Assume também que não foram enfrentados caminhos que gerassem respostas a níveis globais, defende que os programas e implementações objetivando o compromisso com a Agenda se encontram bastante fragmentados e com resultados demasiados díspares (Snedoon et al).

Esta observação delineada por Snedoon et. al (2006), parece ter sido nitidamente apreciada na elaboração dos novos parâmetros da Agenda 2030, em que os objetivos e diretrizes de implementação foram alterados, como exemplo, em relação ao protagonismo dos países subdesenvolvidos, que anteriormente estavam por assim dizer, em um patamar menos restrito de ações dentro dos objetivos da Agenda 21.

Outro aspeto levantado pelos autores e reelaborado nestas diretrizes, consiste no reconhecimento de atuações setoriais, compatibilizando as responsabilidades globais, nacionais e locais e a implantação de dezessete objetivos baseado nos resultados, estudos e lacunas deixadas pela implementação da Agenda 21. (Ministério, 2017, p. 6)

Foi feita nesta investigação, uma analogia desta nova visão do conceito. Este novo olhar para formulação de estratégias para ações que contribuem para o DS, poderia ser comparada com as tradicionais bonecas russas, denominadas Matrioskas. As Matrioskas são bonecas artesanalmente produzidas, que curiosamente escondem dentro de si um segundo exemplar de igual aparência, no entanto, de menor tamanho pois, esta redução nas suas dimensões, permite que essa versão reduzida seja colocada dentro da versão maior. Este processo, de um exemplar da boneca dentro da outra se repete por até sete vezes, conforme figura 1.

Os objetivos relacionados ao DS do planeta, só serão alcançados com a participação de todos os níveis de comunidades e governação existentes.

Neste sentido, a Matrioska do Desenvolvimento Sustentável seria a de no 7, a menor, pois para se chegar a figura última (resultados inerentes de aplicações 
de Políticas Públicas para o DS) seria necessária uma abertura dos diferentes níveis de governação para o encontro dos objetivos finais.

Esta dinâmica da Matrioska se enquadra aos Museus e evidencia o fato dessas instituições serem potenciais agentes contribuidores do DS na fase final do processo no nível local.

O referencial bibliográfico utilizado para elaboração deste estudo demonstra que o conjunto de atores e a multiplicidade de agentes que deverão ter um papel ativo para geração de resultados, definem uma rede complexa. Tendo em conta esta complexidade de redes de atuações, o Planejamento Estratégico se faz protagonista. Quando os objetivos definidos são determinados por pilares de ordem econômica, social e ambiental é possível visualizar diferentes necessidades e soluções que deverão ser contempladas em diferentes âmbitos. Essas diferenças são tanto ao nível global, nacional, regional e local.

Traçar estratégias assertivas para se chegar a um objetivo, é uma das definições mais simplistas do que é um Planejamento Estratégico.

Segundo Rezende na obra Planejamento Estratégico para organizações privadas e públicas", a falta de um pensamento estratégico inviabiliza o processo de Planejamento Estratégico, e cita que este processo exige dos responsáveis pela sua elaboração a capacidade de integrar o conhecimento macro ao micro e vice-versa (Oliveira:1999 apud Rezende,2008, p.3).

Já na obra denominada Texto de Apoio Sobre Planeamento Estratégico (2008, p.9), o autor indica que o conceito pode ser compreendido como um processo ou instrumento a ser utilizado e que o seu uso em Políticas para desenvolvimento de territórios é viável, pois apresenta a característica de reconhecimento de atores, tanto interno e externos facilitando assim o processo de tomada de decisão que assume os objetivos traçados anteriormente. Todo este conjunto de ações integradas tornam os processos de decisão e implementação mais amigáveis e realistas. É de salientar que que 
este processo permite também, visualizar um cenário de possibilidades no período de pré-atuação.

Diante destas definições, já é possível traçar uma relação de dependência entre os movimentos de implementação dos objetivos do DS e o processo de Planejamento Estratégico.

O processo de elaboração de uma Política que contemple estratégias relacionadas ao Desenvolvimento Sustentável, necessita primeiro reconhecer com base nos objetivos delineados pela ONU, quais as deficiências existentes no país que deverão ser tratadas de forma mais enérgica. Com base nisto, o nível de Governo Nacional irá elencar as dificuldades existentes no país e assim traçar um panorama de deficiências e necessidades de melhorias.

Ao traçar o Plano Estratégico, serão delineadas as principais necessidades presentes no país, sugerindo então caminhos genéricos a serem seguidos. Feito isto, são os governos locais que deverão traçar, com base nas suas necessidades e agendas, as iniciativas a serem colocadas nas agendas locais para aprovação política e implantação. Nesta fase, o Planejamento Estratégico deverá ser orientado para uma visão macro das diretrizes da Agenda do Governo Nacional e assim, deverá traçar caminhos assertivos para o processo que se inicia na elaboração, decisão, implantação e avaliação de uma determinada Política Pública local.

$\mathrm{Na}$ obra denominada "Strategic planning as a catalyst for transformative practices" pode ser vista uma segunda visão sobre o que objetiva o Planejamento Estratégico na elaboração de Políticas Públicas territoriais. $O$ autor defende que o Planejamento Estratégico orienta um processo que estimula o encontro de sinergias entre forças de atuação de diferentes âmbitos, setor público, privado, terceiro setor e capital social (Albrechts, 2016, p.6).

Desta forma, o processo de encontro de sinergias, além de complexo tende a ser conflituoso. Considerando este aspeto, Guerra (2000) sintetiza em três pontos os desafios que justificam uma ordem estratégica na implementação 
de Políticas Públicas territoriais, que são: (a) a grande incerteza criada pelos dinamismos do exterior; (b) a crescente complexidade dos processos urbanos; (c) a diversidade dos agentes e interesses que intervêm no progresso urbano $(2000$, p.42).

Em suma, é possível relacionar os pressupostos delineados sobre esta temática na interação entre implementação de Políticas Públicas territoriais somado aos objetivos do DS. Criar espectros de ação que contemplem esses objetivos depende de uma ação mais fragmentada, como exemplo, atribuir e reconhecer aos museus programas dos quais eles estão habilitados a agir. Neste caso, a fragmentação de atores não vem em um sentido pejorativo, mas sim no sentido de reconhecer que há necessidade de uma coordenação de diferentes atores, que nas suas diferentes atribuições deverão contribur nas pretensões relacionadas aos objetivos do DS.

No item seguinte, serão elencadas referências bibliográficas que demonstram a relação existente entre Museus e o DS, justificando assim a necessidade de se conhecer melhor quais são as atribuições destas instituições, que tendem a ser aproveitadas de forma assertiva em programas que objetivam 0 cumprimento dos objetivos do DS.

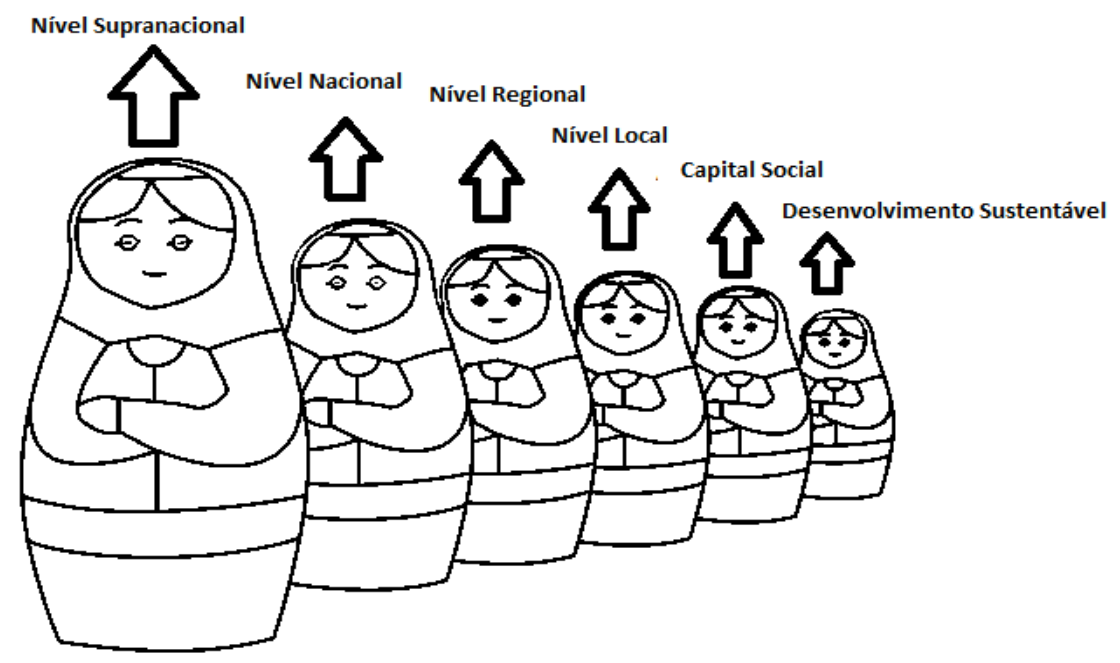

Figura 1: Processo de atuação dos diferentes atores no processo de adequação ao Desenvolvimento Sustentável. Fonte: https://juegos.dibujos.net/matrioskas.html. Edição feita pela autora. 


\section{Museus e Desenvolvimento Sustentável}

Na cimeira ECO`92, realizada no Rio de Janeiro, os Museus adquiriram protagonismo diante dos novos caminhos para o DS dos países. No entanto, este protagonismo esteve reduzido a uma atuação meramente educativa e bastante restrita. Os museus comummente eram reconhecidos como instituições úteis no processo de educação, conscientização e comunicação de ordem meramente ambiental (Boletim ICOM, 2015, p. 10).

Somente no ano de 2007, que o ICOM atribuiu nas definições destas instituições, um contexto mais direcionado a participação dos Museus como prestadores de serviços para a sociedade ${ }^{3}$.

Seguidamente, o próximo movimento que reconhece os museus como impulsionadores do DS está publicado na Culture for agenda 2030, da Organização das Nações Unidas para a Educação, a Ciência e a Cultura (Unesco). Esta publicação é reconhecida como a primeira agenda internacional que reconhece o setor cultural como potencial ferramenta estratégica para o cumprimento dos objetivos da Agenda 2030 da ONU (Unesco ,2018, p. 1).

No entanto, as referências bibliográficas que tratam da relação entre museu e DS localizadas nas plataformas Google Schoolar, b.on e Scopus, são encontradas em maior número nas publicações datadas da segunda década do século XXI. Um exemplo é a obra denominada Sustainable Development Policy do Stewart Museum ${ }^{4}$ que mostra um delineamento dos Princípios, Objetivos e Responsabilidades atribuídas a uma nova Missão do Museu. Esta

\footnotetext{
${ }^{3}$ O Estatuto que confere novas diretrizes aos museus, pode ser consultado no endereço eletrónico http://archives.icom.museum/statutesspa.pdf

${ }^{4}$ Este documento pode ser consultado no endereço eletrónico https://www.stewartmuseum.org/librairie/sustainablepolicy2015/-87.pdf.
} 
Tabela de Políticas ${ }^{5}$ que deverão ser seguidas pela instituição, situado em Montreal, no Canadá, elenca indicadores com base nos objetivos Sociais, Econômicos e Ambiental. Ademais, a Canada Museum Association, em 2010, publicou um guia de diretrizes para orientar os museus na adequação dos parâmetros do DS.

Um segundo exemplo a considerar é o Guia de Boas Páticas para o DS nos Museus da Austrália. Neste documento são elencadas as diretrizes para adequação dos Museus nos conceitos do DS $(2013)^{6}$. Os dois exemplos explicitados, indicam a relevância de organizar parâmetros e diretrizes que permita aos museus demonstrar os outputs gerados que auxiliam num processo de desenvolvimento sustentado da sociedade.

Na obra "Museums: An incubator for sustainable social development and environmental protection", os autores já no início da publicação, defendem que os Museus têm aspetos que se interligam com a dinâmica do D.S. de forma natural, diferente de outras organizações que necessitam de um processo para readequação de suas operações com objetivo de obter resultados sustentáveis nos quesitos de economia, ambiente e sociedade.

Estes fenômenos encontrados nos museus segundo os autores são: “(a) incentivo ao senso coletivo de lugar, memória coletiva, integração social, educação ambiental; (b) mantêm as cidades como centros socioculturais dinâmicos, atuando como instrumentos de coesão entre os diferentes setores da sociedade, considerando a tolerância, democracia e crescimento econômico" (Traduzido pela autora. Gustafon e ljla, 2016:445).

\footnotetext{
${ }^{5}$ O Stewart Museum é apenas um exemplo, pois já existe um processo de aplicação dessas políticas institucionalizada no país.

${ }^{6} \mathrm{O}$ documento foi publicado pela primeira vez em 2002 e passou por revisões em 2013. Pode ser consultado no endereço eletrónico: https://www.amaga.org.au/sites/default/filesuploadedconten/website-content/SubmissionsPolicies/museumsandsustainabilitypolicy2003-2012.pdf.
} 
O reconhecimento destes parâmetros e outros, são organizados em um trabalho fundamental realizado pela $O C D E$ e ICOM, em que as organizações, por intermédio da observação e estudo dos diferentes modelos de museus em diferentes países, organizam indicadores que auxiliam no reconhecimento de atividades exercidas pelas instituições museológicas que geram impacto nos pilares do DS. No entanto, é de suma importância salientar que este Guia tem características bastante genéricas no nível mundial. Por ser um Guia com informação genérica, foi realizado neste estudo um exercício que objetivou reconhecer se os indicadores organizados pela $O C D E$ são aplicáveis no contexto dos museus em Portugal.

Este estudo pretende ser uma contribuição inicial para criação de uma base de indicadores e um Guia para os Museus em Portugal. Está ferramenta intenta facilitar o reconhecimento dos museus como um agente dinamizador no processo de implementação de Políticas Públicas, que objetivam resultados balizados no Conceito do DS.

\section{DESENVOLVIMENTO}

\section{Metodologia}

A escolha da metodologia teve como referência o reconhecimento do problema estudado, a definição de variáveis seguida da recolha, observação e diagnóstico de dados. O problema levantado é a falta de um guia que indique quais são as atribuições conferidas aos museu em Portugal, que correspondem aos objetivos do DS.

Os parâmetros sugeridos no Guia da OCDE (2018) foram a base referencial para realização deste procedimento. A definição de indicadores para análise está fundamenta pelas atividades e Modos de Operação dos Museus em diferentes países, que estão diretamente relacionados aos objetivos da Agenda 2030. Consequentemente, segundo o Guia, estes parâmetros operacionalizados pelos museus, são passíveis de serem utilizados como 
contributos na elaboração de Políticas Públicas que objetivam promover o DS nas localidades, onde o museu está inserido.

O processo de elaboração e organização dos indicadores, na Tabela 1 (disponível no item denominado Resultados), induziu a seguinte hipótese que serviu de base para a definição das variáveis no diagnóstico realizado: Os parâmetros referentes a contribuição dos Museus para o Desenvolvimento Sustentável definidos no Guide for Local Governments, Communities and Museums da OCDE (2018) possibilitam o reconhecimento dos outputs dos museus que contribuem para esta agenda.

Desta forma, o objetivo da hipótese levantada é de identificar se os parâmetros definidos pela publicação da $O C D E$ e ICOM são compatíveis com a mensuração dos outputs gerados pelos museus em Portugal. O processo de indução desta hipótese teve como base o reconhecimento da literatura sobre o tema, que demonstra a necessidade de que parâmetros sejam delineados em conformidade com as necessidades e os processos pró-ativos do país em que o museu está instalado. No entanto, por se tratar de um Guia que teve como base de recolha, observação e análise de dados baseada em diferentes localidades e países, houve a necessidade de identificar se tais parâmetros poderiam ser utilizados de forma genérica em Portugal.

Desta forma, a realização deste estudo está ordenado pelo Plano de Estudo Qualitativo, por meio do procedimento de Estudo de Caso Múltiplo, objetivando a Geração de teoria (ocorrida no processo de indução de hipótese), teoria esta extraída do contexto defendido no Guia da OCDE (2018) e na literatura referenciada no item Revisão de Literatura.

Para esta análise preliminar da relação das instituições museológicas portuguesas e os parâmetros sugeridos no Guia $O C D E$, a amostra dos museus é adotada como variável independente e a presença ou não dos itens sugeridos pela Guia $O C D E$ irá corresponder as variáveis dependentes. 
A operacionalização deste processo seguiu com a recolha de dados com base na aplicação de questionário em dois museus na região centro de Portugal, a saber: (a) Museu Monográfico de Conimbriga e (b) Museu da Águas de Coimbra;

Os parâmetros sugeridos pelo Guia OCDE foram transformados em perguntas que resultou em um questionário simplificado. Os inquiridos deveriam responder Sim ou Não, no entanto, foi considerada a possibilidade do inquirido detalhar sua resposta com um espaço dedicado para isto.

Sendo este um primeiro passo de um processo de investigação, a elaboração do Questionário teve o objetivo apenas de reconhecer se tais atividades, ações ou características eram encontradas nos museus, de forma direta e simplificada.

A escolha de amostras não probabilísticas em um Estudo de caso, segundo Coutinho (2015, p.340) é sempre intencional baseado em critérios teóricos e pragmáticos. A escolha recaiu sobre a amostragem por conveniência e teve como referência as seis modalidades de amostragem de Patton (1980) apontada pela autora, a saber: (a) amostras externas; (b) amostras de casos típicos ou especiais; (c) amostras de variação máxima, adaptada a diferentes condições; (d) amostras de casos críticos; (e) amostras de casos sensíveis ou politicamente importantes; (f) amostras de conveniência (Patton 1989 apud Coutinho, 2015, p. 340), O tipo de amostragem utilizado, segundo Gil (2008, p. 94) é caracterizado pela seleção de elementos com baixo nível de precisão, que possam representar um universo que apresente facilidade de acesso a recolha de dados. Comumente utilizados em estudos de investigação iniciais.

As amostras definidas tiveram como base de escolha uma única região que caracteriza assim um ambiente de estruturas jurídico administrativas com menos disparidades. Outro fator que colaborou para essa escolha está relacionado ao fato do responsável pela elaboração do estudo possuir maior conhecimento das estruturas organizacionais dos Museus situados nesta localidade.

Um importante ponto a salientar é que, quando definido este tipo de seleção das amostras, há um fator que é descartado no resultado do estudo, o 
componente de generalização. Para diminuir esta lacuna existente, a seleção de amostras adotou museus de diferentes temáticas, pois tal característica poderá ser compreendida como uma variável parasita ${ }^{7}$. Como exemplo, em um Museu em que a temática tratada é de Ciências Naturais será possível encontrar uma maior dinâmica nos parâmetros referente aos indicadores pertencentes a classificação ambiental.

Com base na Tabela 1, será feita uma categorização das variáveis com base nas três classificações delineadas pela OCDE (2018). Esta categorização será feita nominalmente.

O resultado da operacionalização deste estudo será delineado no item que segue, denominado Resultados.

\section{Objeto de Estudo: Museu Monográfico de Conimbriga e}

\section{Museu Águas de Coimbra}

A escolha para amostras do Estudo de Caso foram feitas na Região Centro de Portugal situados em Condeixa a Nova, município que pertence ao Distrito de Coimbra, já o segundo Museu da Água de Coimbra está sediado no município de Coimbra, Distrito de Coimbra.

O primeiro, Museu Monográfico de Conimbriga (MMC), tem sua temática baseada nas coleções de achados arqueológicos in situ da Era Romana que segundo evidências, datam "do século IX a.c e Século VII-VIII da nossa era" Nas Figuras 2 e 3 é possível consultar imagens do MMC. A administração do MMC é de responsabilidade da Direção Geral de Cultura de Portugal. Já o

${ }^{7}$ Segundo Rauen variáveis parasitas "são variáveis que, não se constituindo diretamente como objeto de estudo, interferem na relação entre as variáveis independentes e as dependentes" (2012,p.6).

${ }^{8}$ Informação e dados sobre o Museu estão descritos conforme fonte do endereço eletrónico do Museu e Ruínas de Conimbriga. [Acesso em 10 ago. 2019]. Disponível em http://www.conimbriga.gov.pt/portugues/ruinas.html . 
Museu da Água de Coimbra (MAC) é fruto de uma parceria entre a Camâra Municipal de Coimbra e a Empresa Municipal de abastecimento e tratameto de águas da cidade, denominada Águas de Coimbra. A temática nesta instituição é relacionada a contextualização e comunicação da história da utilização dos recursos hídricos da cidade e está sediado em uma antiga estação de captação de água datada de 1922, situado próximo ao Centro Histórico de Cidade, nas margens do Rio Mondego ${ }^{9}$. Nas Figuras 4 e 5 é possível consultar imagens do MAC.

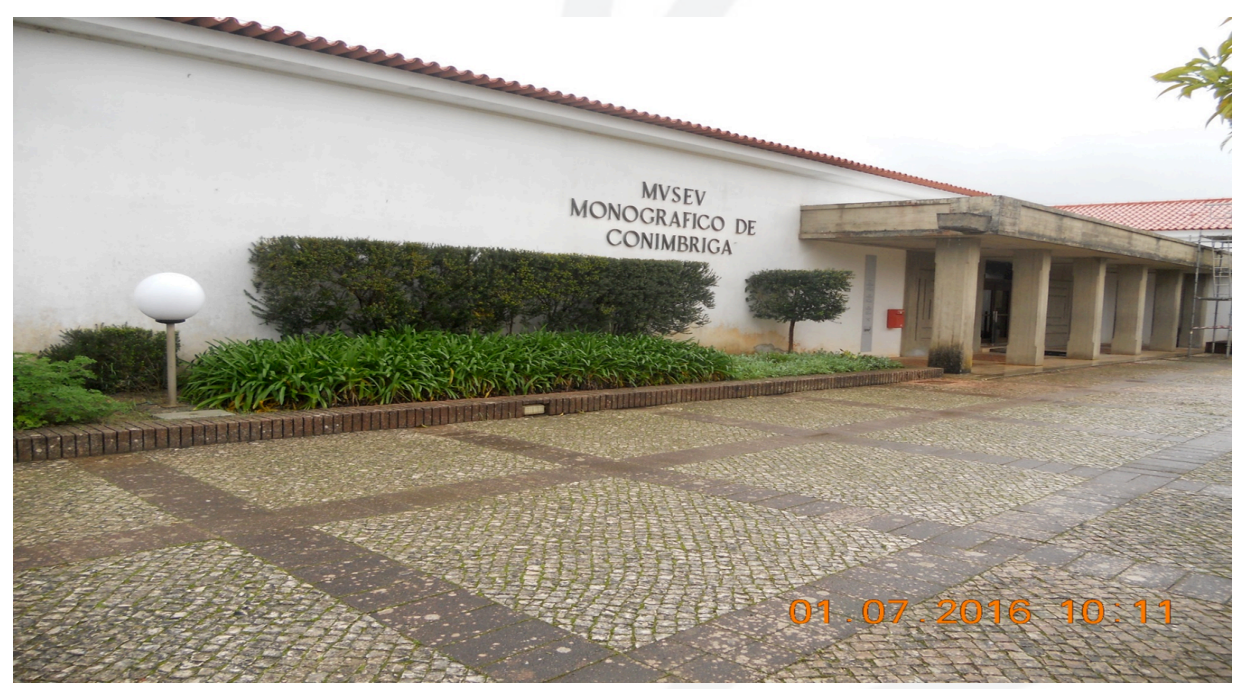

Figura 2: Fachada da entrada do Museu Monográfico de Conimbriga. Fonte: Arquivo pessoal

${ }^{9}$ Fonte de informação: endereço eletrônico Roteiro dos Museus e Espaços Museológicos da Região Centro de Portugal. [Acesso em 10 de ago. 2019] Disponível em: http://roteiromuseus.ccdrc.pt/museusficha.aspx?idMuseu=57\&tipologia=4 


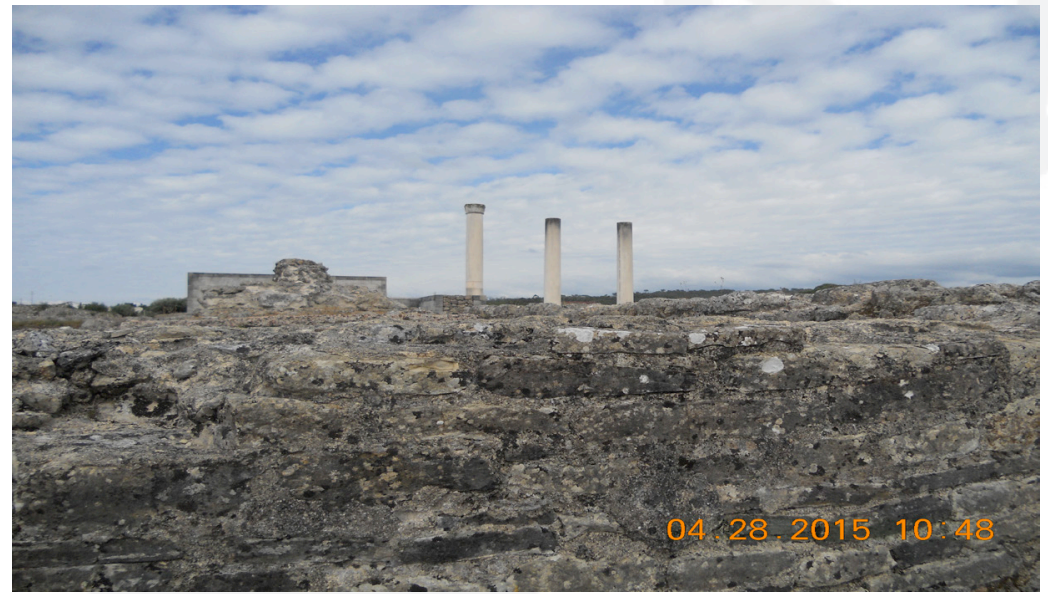

Figura 3: Vista da entrada das ruínas Romanas de Conimbriga. Fonte Arquivo Pessoal.

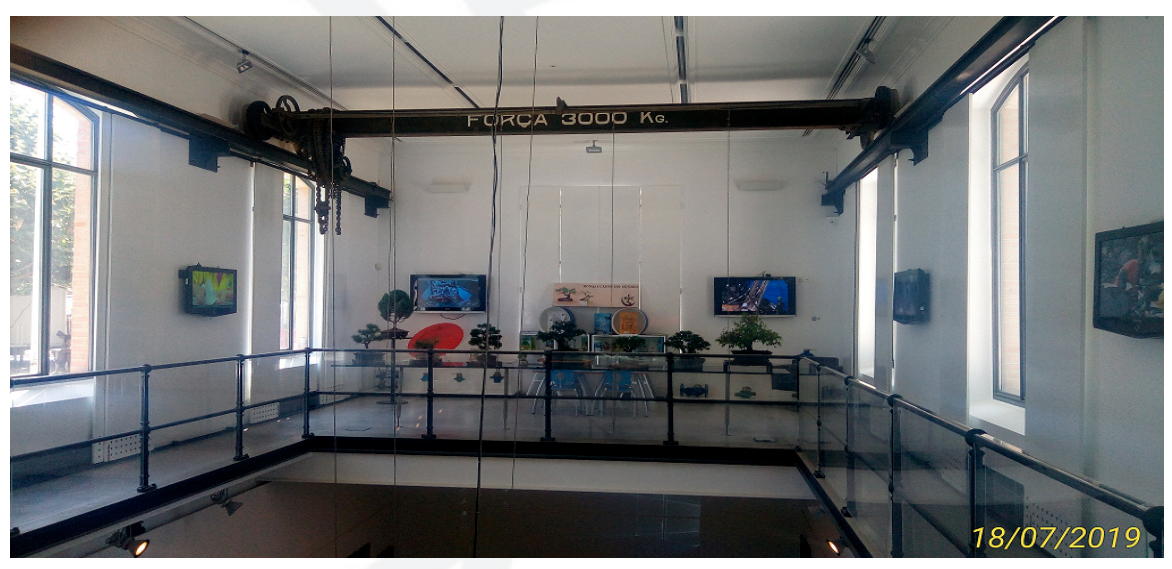

Figura 4: Vista interna do espaço expositivo do Museu Águas de Coimbra. Fonte: Arquivo pessoal. 


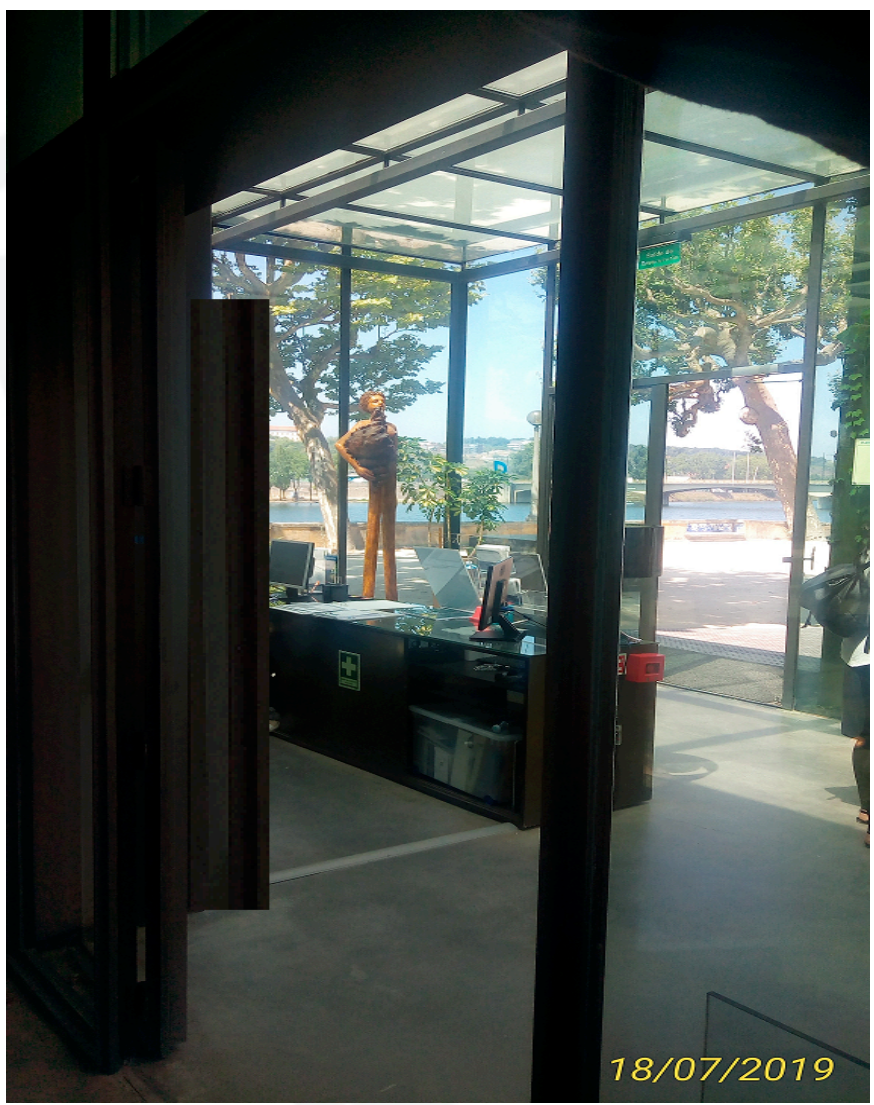

Figura 5: Vista da entrada e recepção do Museu Águas de Coimbra. Vista da Margem do Rio Mondego. Fonte: Arquivo pessoal.

\section{RESULTADOS}

Neste item serão mostrados os resultados advindos da recolha de dados nos museus. Diante do número de museus inquiridos, os dados para análise formam uma base reduzida. No entanto, é possível identificar outputs gerados nos Museus que correspondem aos parâmetros sugeridos pela OCDE.

O tratamento de dados considerou que: (a) as resposta positivas receberiam a pontuação 1 ao indicador em questão; (b) já as respostas negativas, a pontuação atribuída é igual a 0.

No indicador no 7, Classificação Ambiental, relacionado a existência de transporte limpo as respostas foram negativas em ambos os casos, e foram 
representadas como nulas.

Depois de organizados os dados iniciais do estudo, o tratamento de dados seguiu com a identificação do número de ocorrência de cada grupo de indicadores. A totalização de ocorrências positivas de cada grupo de indicadores serviram de base para análise do nível de sustentabilidade de cada Museu.

O primeiro resultado demonstrado no Gráfico 1 é referente ao número de respostas positivas recebidas em cada um dos três grupos de indicadores do DS dos dois Museus.

Com base nestes resultados, o estudo segue para o penúltimo item denominado Análise de dados, em que serão demonstradas reflexões sobre os resultados observados no que se refere ao reconhecimento do Museu como agente dinamizador do DS. Somado a isto será apresentado também, um breve diagnóstico para demonstrar como se relacionam os indicadores da OCDE com os objetivos da Agenda 2030.

Tabela 1: Organização de Parâmetros e Indicadores do Desenvolvimento Sustentável em Museu

\begin{tabular}{|l|l|l|}
\hline \multicolumn{1}{|c|}{ ECONÔMICO } & \multicolumn{1}{|c|}{ SOCIAL } & \multicolumn{1}{c|}{ AMBIENTE } \\
\hline $\begin{array}{l}\text { 1.Criação de } \\
\text { empregos }\end{array}$ & 1.Regeneração urbana & $\begin{array}{l}\text { 1.Criar programas de } \\
\text { conscientização de } \\
\text { preservação do meio } \\
\text { ambiente }\end{array}$ \\
\hline 2.Contribuição no PIB & $\begin{array}{l}\text { 2.Atuar no controle de } \\
\text { gentrificação }\end{array}$ & $\begin{array}{l}\text { 2.Criar programações e } \\
\text { roteiros temáticos } \\
\text { sobre otema }\end{array}$ \\
\hline 3.Contribuição Fiscal & $\begin{array}{l}\text { 3.Atuar como dinamizador em } \\
\text { busca de soluções e PP nos } \\
\text { centros de convivência das } \\
\text { localidades de }\end{array}$ & $\begin{array}{l}\text { 3.Readequar } \\
\text { contribuir edifícios de } \\
\text { eficiência energética }\end{array}$ \\
\hline $\begin{array}{l}\text { 4.Start ups e/ou } \\
\text { incubadoras } \\
\text { associadas ao museu }\end{array}$ & $\begin{array}{l}\text { 4.Servir de elo entre } \\
\text { universidade e população para } \\
\text { regeneração de edifícios } \\
\text { degradados de valor cultural }\end{array}$ & $\begin{array}{l}\text { 4.Contribuir para a a diminuição de uso dos } \\
\text { produtos plásticos e } \\
\text { descartáveis }\end{array}$ \\
\hline $\begin{array}{l}\text { 5.Facilitar e dinamizar } \\
\text { o empreendedorismo }\end{array}$ & $\begin{array}{l}\text { 5.Atuar como organizador de } \\
\text { reuniões entre sociedade e } \\
\text { Poder Político na busca de } \\
\text { Políticas de organização urbana }\end{array}$ & $\begin{array}{l}\text { 5.Contribuir para } \\
\text { diminuição de uso de } \\
\text { tinteiros e papel de } \\
\text { impressão }\end{array}$ \\
\hline
\end{tabular}




\begin{tabular}{|l|l|l|}
\hline $\begin{array}{l}\text { 6.Colocar a localidade } \\
\text { como uma opção para } \\
\text { alocação de empresas }\end{array}$ & $\begin{array}{l}\text { 6.Oferta de cursos e work shops } \\
\text { relacionados aos assuntos de } \\
\text { interesse da sociedade }\end{array}$ & $\begin{array}{l}\text { 6.Considerar uso de } \\
\text { papel reciclável em } \\
\text { legendas, produção de } \\
\text { materiais e outros }\end{array}$ \\
\hline $\begin{array}{l}\text { 7.Profissionalização } \\
\text { do turismo - relação } \\
\text { das prestadoras de } \\
\text { serviço com os } \\
\text { museus }\end{array}$ & $\begin{array}{l}\text { 7.Criar serviços educativos de } \\
\text { vários níveis - dando atenção } \\
\text { ao público sénior }\end{array}$ & $\begin{array}{l}\text { 7.Criar quando possível, } \\
\text { serviços de transportes } \\
\text { menos poluentes para } \\
\text { dar acesso aos museus }\end{array}$ \\
\hline & $\begin{array}{l}\text { 8.Catalisador de atividades } \\
\text { criativas }\end{array}$ & \\
\hline
\end{tabular}

Gráfico 1: : Comparação entre os dois Museus analisados com base no Parâmetros de Classificação

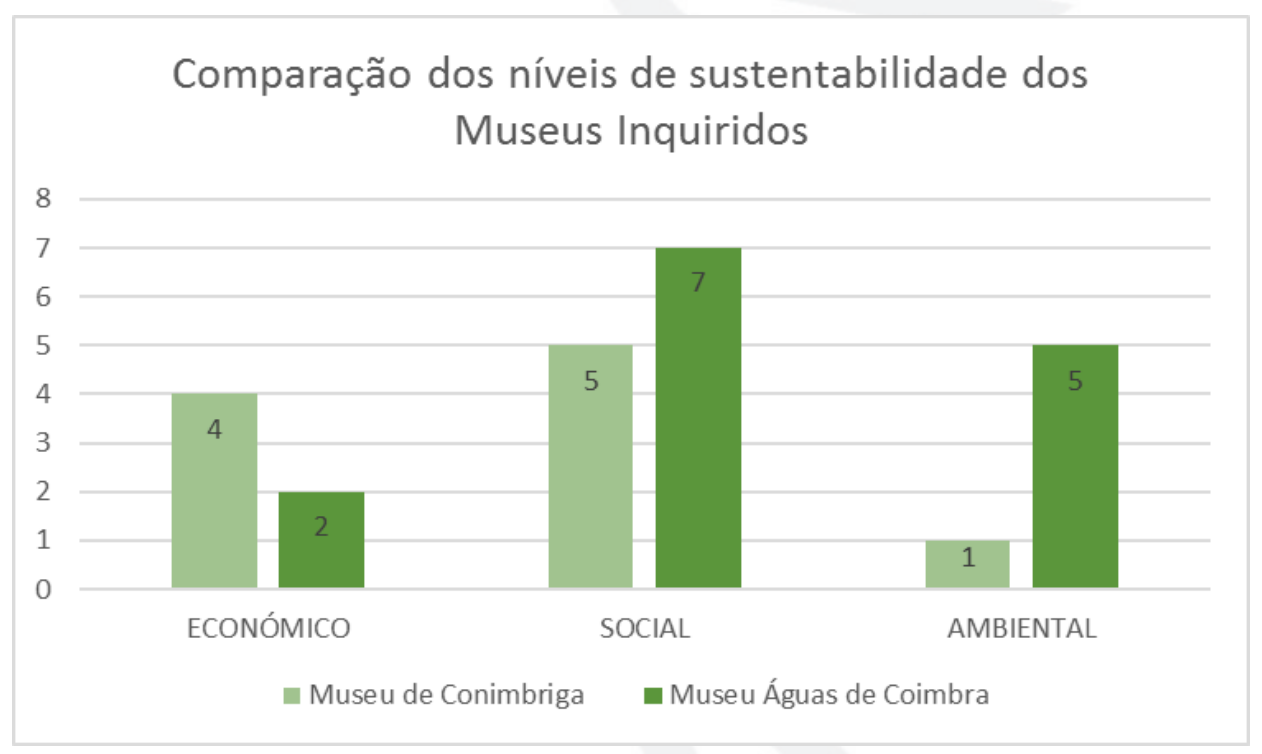

\section{ANÁLISE DE RESULTADOS}

Os resultados obtidos na recolha de dados dos museus, demonstram que ambas instituições reúnem outputs que poderão ser considerados como contributos para o DS. No entanto, é possível notar uma diferença entre os níveis de resposta quando comparado os três conjuntos de indicadores analisados. Esta diferença poderá ser consequência de uma variável parasita que se trata da temática do Museu. 
Em consulta ao Gráfico 1 é possível verificar que o MAC, que tem um apelo ambiental, responde com maior intensidade ao grupo de indicadores ambientais. Já o MMC, por ter um apelo turístico mais relevante, demonstra um resultado mais intenso no aspeto econômico.

As informações obtidas demonstram a efetiva possibilidade de utilizar estes parâmetros para reconhecer as aptidões de cada instituição e ter assim uma massa crítica de dados capaz de demonstrar ao Poder Público, como os Museus podem atuar de forma assertiva nas formulação de estratégias para o atendimento da Agenda 2030 da ONU.

Para ilustrar como os resultados gerados pelos Museus respondem aos dezessete objetivos da Agenda 2030, esta Análise de Resultados acresce um breve trabalho realizado de correlação entre os indicadores organizados com base nos Parâmentros do Guia da OCDE e os dezessete objetivos para o DS estabelecidos pela ONU aos países signatários.

Este trabalho de correlação teve como referência o "Relatório nacional sobre a implementação da Agenda 2030 para o DS de Portugal".

O Gráfico 2 demonstra os resultados advindos desta análise de correlação que considerou os dezesseste objetivos da ONU. Os resultados são advindos da aplicação do seguinte método: (a) quando reconhecida uma correlação entre um indicador e os objetivos da Agenda 2030, o Grupo deste indicador receberá a pontuação 1; (b) quando está correlação não for percebida deverá então ser atribuída a pontuação 0 .

A análise de correlação realizada demonstra que os indicadores dos Museus atendem aos dezessete objetivos do DS. A correlação entre os grupos de indicadores são díspares, no entanto, não houve nenhum caso em que todos os grupos dos indicadores responderam negativamente aos objetivos da Agenda 2030. 
Gráfico 2: Correlação entre os indicadores da OCDE e os objetivos da Agenda 2030-ONU

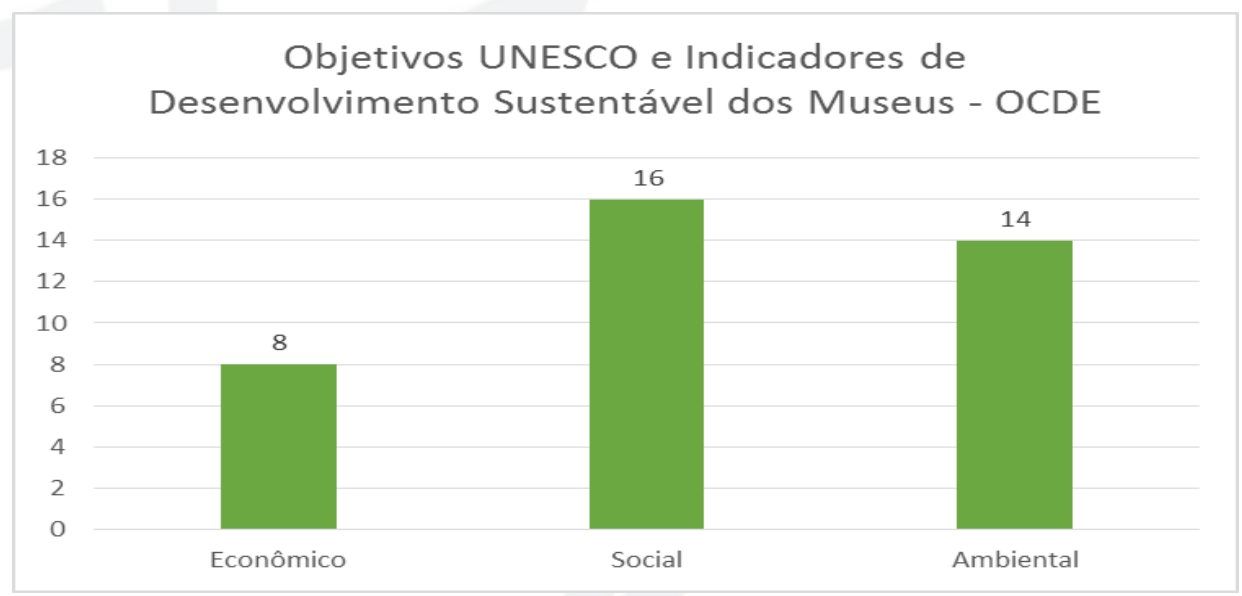

\section{CONSIDERAÇÕES FINAIS}

O estudo realizado demonstrou resultados satisfatório para o objetivo da proposta delineada neste trabalho.

Foi possível reconhecer que é viável utilizar os parâmetros definidos pela OCDE para aferir os níveis de resposta dos museu para cada pilar do DS. No entanto, este estudo é apenas uma prévia do que uma investigação mais aprofundada poderá resultar. Ao considerar a realização do Planejamento Estratégico como instrumento para cumprir os objetivos do DS, é possível visualizar, já nestes resultados, quais são os pontos estratégicos que os Museus poderão contribuir de forma assertiva para obtenção de resultados satisfatórios.

Uma outra forma de utilizar estes resultados é o Museu divulgar ao Poder Público o seu nível de resposta diante de objetivos relacionados ao DS, que por consequência agrega à sociedade uma melhor condição de vida e também, contribui para a redução de disparidades entre a população, incentivando uma atuação mais representativa do capital social do microambiente em que o museu está instalado.

Um terceiro ponto relevante, é a possibilidade de criar um Guia de Boas Práticas que irá permitir aos Museus em Portugal, ter uma base referencial para 
implementar na organização tais práticas ou mesmo, melhorar as atividades e modos de operação no que diz respeito aos seus níveis de sustentabilidade.

No entanto, como já descrito, este é um contributo inicial que deverá ser desenvolvido com maior complexidade. Resultados que poderão ser buscados em estudos futuros estão relacionadas a: (1) um estudo com aplicação de método de seleção de amostras que garanta a generalização dos resultados; (b) estudo de indicadores internacionais de sustentabilidade aplicados aos museus que possam servir de referência para a construção de um conjunto de indicadores customizados para o caso Português ou outro país de interesse; (c) Definição de nível desejado mínimos e máximos de sustentabilidade; (d) Recolha de dados realizada com diferentes métodos.

\section{AGRADECIMENTO}

Este trabalho recebeu o gentil apoio do Diretor do Museu Monográfico de Conimbriga, José Ruivo e da Coordenadora do Museu Águas de Coimbra, Ana Santos e tutoria dos Professores do Departamento de Ciências Sociais e Políticas do Território da Universidade de Aveiro - Professor Doutor Artur Rosa Pires e Professor Doutor José Manuel Martins.

\section{BIBLIOGRAFIA}

Albrechts, L. (2016). Strategic planning as a catalyst for transformative practices. Publicado em Haselsberger, B. (Ed) Encounters in Planning Thought. 16 Autobiographical Essays from Key Thinkers in Planning. Routledge, New York.

Boletim ICOM Portugal. (2015). 3rd ed. [ebook] Ana Carvalho. [Acesso em 18 dez.2018]. Disponível em <http://icomportugal.org/multimedia/Boletim\%20ICOM\%20Portugal\%2 0s\%C3\%A9rie\%20III\%20n_\%C2\%BA\%203\%20Maio\%202015.pdf>

Coutinho, Clara P. (2015). Metodologia de Investigação em Ciências Sociais e Humanas: Teoria e Prática. 2nd ed. Coimbra: Almedina.

Canadian Museums Association. (2010). A Sustainable Development Guide for Canada"s Museums. 
Gil, A. (2008). Métodos e técnicas de pesquisa social. 6th ed. São Paulo: Atlas.Gil

Gustafsson, C. and ljla, A. (2016). Museums: An incubator for sustainable social development and environmental protection. International Journal of Development and Sustainability

Ministério dos Negócios Estrangeiros (2017). Relatório nacional sobre a implementação da Agenda 2030 para o Desenvolvimento Sustentável Portugal. Nova York: Vitorino Mello Oliveira e Francisca Navega. [Acesso em 12 out.2018]. Disponível em:

$<$ https://sustainabledevelopment.un.org/content/documents/15771Por tugal2017_PT_REV_FINAL_28_06_2017.pdf>

Morais, C. (2004). Escalas de Medida, Estatística Descritiva e Inferência Estatística. 1st ed. Bragança.

Museums and Sustainability (2001): Guidelines for Policy and Practice in Museums and Galleries' was initiated at the Annual General Meeting of Museums Australia.

Nascimento, E. (2012). Trajetória da sustentabilidade: do ambiental ao social, do social ao econômico. Estudos Avançados, 26(74), 51-64. [Acesso em 20 de dez. 2018].

Nosso futuro comum. (1991). Rio de Janeiro: Fundação Getúlio Vargas.

OCDE and ICOM (2018). CULTURE AND LOCAL DEVELOPMENT: MAXIMISING

THE IMPACT -Launch version Guide for Local Governments, Communities and Museums. 1st ed. [ebook] Conselho Internacional de Museus e Organização para a Cooperação e Desenvolvimento Econômico.

Rauen, Fábio José (2012). Pesquisa Científica: Discutindo a Questão Das Variáveis. Publicado nos Anais do IV Simpósio sobre Formação de Professores - SIMFOP da Universidade do Sul de Santa Catarina, Campus de Tubarão, de 7 a 11 de maio de 2012, pp.1-14.

Rezende, D. Alcides (2008). Planejamento de sistemas de informação e informática. São Paulo: Editora Atlas S.A.

Santos, M. (2008). Texto de Apoio Sobre Planeamento Estratégico. 1st ed. Évora.

Sneddon, C., Howarth, R. and Norgaard, R. (2006). Sustainable development in a post-Brundtland world. Ecological Economics, 57(2), pp.253-268.

UNESCO - United Nations Educational, Scientific and Cultural Organization (2018). Culture for the 2030 Agenda. Sustainable Development Goals. 
[online] Paris: United Nations Educational, Scientific and Cultural Organization, pp.3-45.

Wickham, M. and Lehman, K. (2015). Communicating sustainability priorities in the museum sector. Journal of Sustainable Tourism, 23(7), pp.10111028. 